\title{
Modelling the dynamics of dengue real epidemics
}

\author{
Claudia P. Ferreira \\ Depto de Bioestatística, IB, UNESP \\ E-mail: pio@ibb.unesp.br \\ Suani T.R. Pinho \\ Instituto de Física, Universidade Federal da Bahia \\ E-mail: suani@ufba.br. \\ Lourdes Esteva \\ Facultad de Ciencias, Universidad Nacional Autónoma de México \\ E-mail: esteva@lya.fciencias.unam.mx. \\ F. R. Barreto, V. C. Morato e Silva, M. G. L. Teixeira \\ Instituto de Saúde Coletiva, Universidade Federal da Bahia \\ E-mail: florisneide@ufba.br, morato@gmail.com, magloria@ufba.br
}

Resumo: We apply a mathematical model for dengue transmission that comprises the mosquito, the human population and control mechanisms applied to the mosquito population to study two epidemics dengue situations that occurred at Salvador, Brazil at 1995 and 2002. Using real data, we are able to determine the basic reproductive number of the epidemics, $R_{0}$, as well as analysis its time evolution, $R(t)$, during the epidemics. We shown that control mechanism applied only on the adult mosquito form are not sufficient to stop dengue transmission, emphasizing the importance of the control applied on the aquatic mosquito phase.

\section{Introduction}

The infectious diseases are still a relevant problem for human life. Nowadays, due to the intense flow of people around the world and within the cities, the understanding of their complex dynamics is a multidisciplinary issue. Concerning dengue, a vector transmitted disease, there is no vaccine against any of the four serotypes of the virus, although many efforts are done in that direction. The modelling of the dynamics of dengue may be very helpful for testing both the adopted vector control strategies $[3,9,10]$ and the action of future vaccines.

In South and North America, there are records of occurrence of all serotypes of dengue virus, while in Brazil, until now, only 3 serotypes (DENV1, DENV2 and DENV3) have been reported. However, Brazil is responsible for $80 \%$ of dengue cases in South America. In order to investigate comparatively the action of vector control on the dynamics of dengue, we analyze two outbreaks of dengue disease occurred at Salvador, Bahia, Brazil in 1995 and 2002. In the first outbreak only DENV2 was circulating, and no vector population control was been applied by the local health authorities. Also, the population was completely susceptible for this serotype. On the other hand, during the second outbreak, the prevalent serotype was DENV3 and mosquito control was in course.

In this work we use a mathematical model [7] to analyze those dengue outbreaks. Our focus is on the calculation of the basic reproductive number, $R_{0}$, of actual epidemics as well as on the analysis of its time evolution $R(t)$ since it changes during the epidemics. There are different ways to estimate $R_{0}$ for vector transmitted diseases using actual data of epidemics [4]. Another important feature that we take into account for our analysis is the variation of entomological 
parameters with the temperature [8]. Our aim is to estimate the role of vector control in the reduction of the intensity and duration of the epidemics.

This work is organized as follow. In Section 2 we formulate the model. In Section 3 we obtain the disease free equilibrium, we calculate the expression for $R_{0}$, and its values for the 1995-1996

and 2002 epidemics in Salvador. In Section 4, we calculate and discuss the time dependence of $R_{0}$ as well as its behavior for actual epidemics in Salvador in 1995-1996 and 2002. Finally, Section 5 closes this paper with concluding remarks and perspectives.

\section{Formulation of the model}

The model developed here is based upon the one given in [7], where the mosquito population, $M$, is divided into four compartments: aquatic $(A)$, susceptible $\left(M_{s}\right)$, exposed $\left(M_{e}\right)$, and infectious $\left(M_{i}\right)$, being the three last classes related to the winged female form of the mosquito. The entomological parameters are: the intrinsic oviposition rate, $\delta$; the per capita mortality rate of adult female and aquatic forms, $\mu_{a}, \mu_{m}$, respectively; and the per capita rate at which mosquitoes emerge from the aquatic phase and become female adults, $\gamma_{m}$. The remaining parameters are the carrying capacity, $C$, the fraction of female mosquitoes hatched from eggs, $k$, with $0<k<1$, and control efforts, modeled by additional mortality rates applied to the aquatic and terrestrial phase, respectively, $c_{a}$ and $c_{m}$.

The human population, $H$, is assumed to be constant with per capita mortality rate given by $\mu_{h}$, and it is divided as susceptible $\left(H_{s}\right)$, exposed $\left(H_{e}\right)$, infectious $\left(H_{i}\right)$, and recovered $\left(H_{r}\right)$ individuals.

Flows from susceptible to infected class of both populations depend on the biting rate of the mosquitoes, the transmission probabilities, as well as the number of infectives and susceptibles of each species. The per capita biting rate of mosquitoes, $b$, is the average number of bites per mosquito per day, while the transmission probability is the probability that an infectious bite produces a new case in a susceptible member of the other species. We denote by $\beta_{h}$, and $\beta_{m}$ the transmission probabilities from mosquito to human, and human to mosquito, respectively. Assuming that $b M / H$ is the average number of bites that a human received, the infection rates per susceptible human, and susceptible vector are given, respectively, by

$$
b \beta_{h} \frac{M}{H} \frac{M_{i}}{M}=\frac{b \beta_{h}}{H} M_{i} \quad \text { and } \quad \frac{b \beta_{m}}{H} H_{i}
$$

We assume that the population of exposed (infected by not infectious) humans, and mosquitoes become infectious at a rate $\theta_{h}$, and $\theta_{m}$, respectively, being the reciprocal quantities $1 / \theta_{h}$ and $1 / \theta_{m}$ the intrinsic and extrinsic periods of virus replication in humans and mosquitoes, respectively. In the case of the mosquitoes, the extrinsic period depends on the temperature [5]. In fact, higher temperatures reduce $\theta_{m}$ by increasing the virus replication rate and intensify the efficiency of the vector in transmitting dengue by increasing the number of blood meals during a gonotrophic cycle [13]. Finally, the humans recover from the disease at a per capita constant rate $\alpha_{h}$, where $1 / \alpha_{h}$ is the infectious period, meanwhile the mosquitoes remain infectious during their entire life.

Since $H_{r}=H-H_{s}-H_{i}$, is enough to consider the system in the variables $A, M_{s}, M_{e}, M_{i}, H_{s}, H_{i}$. Therefore, the dynamical system is given by

$$
\begin{aligned}
\frac{d A}{d t} & =k \delta(t)(1-A / C) M-\left(\gamma_{m}(t)+\mu_{a}(t)+c_{a}(t)\right) A \\
\frac{d M_{s}}{d t} & =\gamma_{m}(t) A-\frac{b \beta_{m} M_{s} H_{i}}{H}-\left(\mu_{m}(t)+c_{m}(t)\right) M_{s} \\
\frac{d M_{e}}{d t} & =\frac{b \beta_{m} M_{s} H_{i}}{H}-\left(\theta_{m}(t)+\mu_{m}(t)+c_{m}(t)\right) M_{e}
\end{aligned}
$$




$$
\begin{aligned}
\frac{d M_{i}}{d t} & =\theta_{m}(t) M_{e}-\left(\mu_{m}(t)+c_{m}(t)\right) M_{i} \\
\frac{d H_{s}}{d t} & =\mu_{h}\left(H-H_{s}\right)-\frac{b \beta_{h} H_{s} M_{i}}{H} \\
\frac{d H_{e}}{d t} & =\frac{b \beta_{h} H_{s} M_{i}}{H}-\left(\theta_{h}+\mu_{h}\right) H_{e} \\
\frac{d H_{i}}{d t} & =\theta_{h} H_{e}-\left(\alpha_{h}+\mu_{h}\right) H_{i} .
\end{aligned}
$$

We remark that the mosquito entomological parameters $\delta(t), \gamma_{m}(t), \mu_{a}(t), \mu_{m}(t)$ and $\theta_{m}(t)$ varying with the daily temperature, and therefore, are functions of the time $t$. In particular, higher temperatures increase the mosquito's survival and oviposition rate and accelerate its reaching to the adult phase. Likewise, rainfall has a positive influence on mosquito breeding conditions, creating and maintaining natural and artificial oviposition containers [13]. Also, the parameters $c_{a}(t)$ and $c_{m}(t)$ are function of the time, since the mosquito control is primarily made during the favorable period for dengue epidemics outbreak. The rest of the parameters are maintained constant. Moreover, the entomological parameters values considered in this work was estimated using the results described at [8] (Tables 4, 5, 8, 10 of [8]). For temperature values not contemplated in these Tables we applied a linear interpolation to evaluate the mosquito parameters. In short, variables and parameters range are summarized in Table 1.

Tabela 1: Parameters used in the model, biological description and range of values $[7,8, ?, 12,5]$.

\begin{tabular}{lll}
\hline Parameter & Biological meaning & Range of values \\
\hline$\delta$ & average oviposition rate & $0-11.2$ days $^{-11 *}$ \\
$\mu_{m}$ & average mosquito mortality rate & $0.02-0.09$ days $^{-1} 2 *$ \\
$\mu_{a}$ & average aquatic mortality rate & $0.01-0.47$ days $^{-1} 3 * *$ \\
$\gamma_{m}$ & average aquatic transition rate & $0-0.19$ days $^{-1} 4 * *$ \\
$\theta_{m}$ & extrinsic incubation & $0.02-0.2$ days $^{-1} 5 * * *$ \\
$\mu_{h}$ & human mortality rate & $0.0143-0.0167$ years $^{-1}$ \\
$\theta_{h}$ & intrinsic incubation rate & $0.083-0.17$ days $^{-1}$ \\
$\alpha_{h}$ & recovering rate & $0.083-0.25$ days $^{-1}$ \\
$k$ & mosquito sex ratio & $0-1$ \\
$C$ & carrying capacity & 2 mosquitoes per person \\
$b$ & average bit per mosquito per day & $0-1$ \\
$\beta_{m}$ and $\beta_{h}$ & effective contact rates & 0.75 \\
$c_{a}$ and $c_{m}$ & control effort rates & $0-1$ \\
\hline
\end{tabular}

* for the temperature $T$ in the interval of $T \in[10.54,33.41]{ }^{\circ} \mathrm{C}, * *$ for $T \in[10,40.6]{ }^{\circ} \mathrm{C}$ and $* * *$ for $T \in[11,36]^{\circ} \mathrm{C}$.

\section{Basic Reproductive Number}

\subsection{Disease-free equilibrium}

In order to obtain the equilibrium points of system (1), and an expression for $R_{0}$ at the beginning of the epidemic, we assume that the entomological parameters $\delta(t), \gamma_{m}(t), \mu_{a}(t), \mu_{m}(t), \theta_{m}(t)$ and the control efforts given by $c_{a}(t)$ and $c_{m}(t)$ are constant. Therefore, the disease-free equilibrium is $E_{0}=\left(\bar{A}, \bar{M}_{s}, 0,0, H, 0,0\right)$, where $\bar{A}$ and $\bar{M}_{s}$ are the positive solution of the algebraic system

$$
\begin{aligned}
& 0=k \delta(1-A / C) M-\left(\gamma_{m}+\mu_{a}+c_{a}\right) A \\
& 0=\gamma_{m} A-\left(\mu_{m}+c_{m}\right) M_{s}
\end{aligned}
$$


given by

$$
\bar{A}=\frac{C}{\left(\mu_{m}+c_{m}\right)}\left(1-\frac{1}{R_{M}}\right), \quad \bar{M}_{s}=\frac{\gamma_{m} \bar{A}}{\left(\mu_{m}+c_{m}\right)} .
$$

In the expression above $R_{M}$ denotes the "basic offspring" of the mosquito population which is given by

$$
R_{M}=\frac{k \delta \gamma_{m}}{\left(\mu_{m}+c_{m}\right)\left(\gamma_{m}+\mu_{a}+c_{a}\right)} .
$$

We note that the condition $R_{M}>1$ is necessary in order to have a positive population of mosquitoes.

\subsection{Epidemic and Endemic equilibrium}

Using the next generation operator approach $[2,6]$, we compute the basic reproductive number $R_{0}$ associated with the disease-free equilibrium. The non-negative matrix, $K$, of the infection terms, and the non-singular M-matrix, $T$, of the transition terms are given, respectively, by

$$
K=\left(\begin{array}{rrrr}
0 & 0 & 0 & \frac{b \beta_{m} \bar{M}_{s}}{H} \\
0 & 0 & 0 & 0 \\
0 & b \beta_{h} & 0 & 0 \\
0 & 0 & 0 & 0
\end{array}\right)
$$

and

$$
T=\left(\begin{array}{rrrr}
\theta_{m}+\mu_{m}+c_{m} & 0 & 0 & 0 \\
-\theta_{m} & \mu_{m}+c_{m} & 0 & 0 \\
0 & 0 & \theta_{h}+\mu_{h} & 0 \\
0 & 0 & -\theta_{h} & \mu_{h}+\alpha_{h}
\end{array}\right) .
$$

In $[2,6]$ it is shown that the basic reproductive number, $R_{0}$, is equal to the spectral ratio of the matrix $K T^{-1}$. Recall that the spectral ratio of a matrix is equal to its larger eigenvalue, we obtain that the eigenvalues of $K T^{-1}$ are zero of multiplicity two, and

$$
\pm \sqrt{\frac{\theta_{h} \theta_{m} b^{2} \beta_{h} \beta_{m}}{\left(\theta_{h}+\mu_{h}\right)\left(\theta_{m}+\mu_{m}+c_{m}\right)\left(\alpha_{h}+\mu_{h}\right)\left(\mu_{m}+c_{m}\right)} \frac{\bar{M}_{s}}{H}} .
$$

Therefore,

$$
R_{0}=\sqrt{\frac{\theta_{h} \theta_{m} b^{2} \beta_{h} \beta_{m}}{\left(\theta_{h}+\mu_{h}\right)\left(\theta_{m}+\mu_{m}+c_{m}\right)\left(\alpha_{h}+\mu_{h}\right)\left(\mu_{m}+c_{m}\right)} \frac{\bar{M}_{s}}{H}} .
$$

Observe that $R_{0}$, the reproduction number of dengue, depends on the mosquito and human vital parameters, on the fraction between the susceptible mosquito and the total human population size, and also on the product of the transmission coefficients and the square of mosquitoes biting rate, $b^{2} \beta_{h} \beta_{m}$, emphasizing that a new case of dengue can occur only after two bites from the same mosquito. On the other hand, we also observe that dengue control appears explicitly in the expression for $R_{0}$, only on the terrestrial phase, $c_{m}$. However, control efforts applied on the mosquito aquatic phase decreases mosquito offspring number (equation 3 ) resulting in a decrease of mosquito susceptible population.

\subsection{Estimation of $R_{0}$ for actual epidemics}

Following [4], we assume that at the beginning of the epidemic, the cumulative number of cases, $K$, vary like $K \propto \exp (\Lambda t)$, where $\Lambda$ is the force of infection. In this case, the number of infectious and exposed vectors and the number of infectious and exposed host vary similar:

$$
\begin{aligned}
M_{e}(t) & \sim M_{e_{0}} \exp (\Lambda t), \\
H_{e}(t) & \sim H_{e_{0}} \exp (\Lambda t), \\
M_{i}(t) & \sim M_{i_{0}} \exp (\Lambda t), \\
H_{i}(t) & \sim H_{i_{0}} \exp (\Lambda t),
\end{aligned}
$$


where $M_{e_{0}}, H_{e_{0}}, M_{i_{0}}$, and $H_{i_{0}}$ are constants. Further, the number of non-susceptible hosts and vectors can be assumed negligible, and

$$
\begin{aligned}
M_{s}(t) & =\bar{M}_{s} \text { and } \\
H_{s}(t) & =H .
\end{aligned}
$$

Substituting equations (5) and (6) in the expression for the derivative of $M_{e}, H_{e}, M_{i}$ and $H_{i}$ (system 1) we obtain

$$
\begin{aligned}
{\left[\frac{\Lambda}{\left(\theta_{m}+\mu_{m}+c_{m}\right)}+1\right] M_{e_{0}} } & =\frac{b \beta_{m} \bar{M}_{s}}{\left(\theta_{m}+\mu_{m}+c_{m}\right) H} H_{i 0} \\
{\left[\frac{\Lambda}{\left(\theta_{h}+\mu_{h}\right)}+1\right] H_{e_{0}} } & =\frac{b \beta_{h}}{\left(\theta_{h}+\mu_{h}\right)} M_{i 0} \\
{\left[\frac{\Lambda}{\left(\mu_{m}+c_{m}\right)}+1\right] M_{i_{0}} } & =\frac{\theta_{m}}{\left(\mu_{m}+c_{m}\right)} M_{e 0} \\
{\left[\frac{\Lambda}{\left(\alpha_{h}+\mu_{h}\right)}+1\right] H_{i_{0}} } & =\frac{\theta_{h}}{\left(\alpha_{h}+\mu_{h}\right)} H_{e_{0}} .
\end{aligned}
$$

Multiplying the right and left hands of the above equations and using the definition of $R_{0}$ given by equation (4) leads to the following relation between the basic reproductive number, $R_{0}$, and the force of infection, $\Lambda$ :

$$
R_{0}^{2}=\left(\frac{\Lambda}{\theta_{m}+\mu_{m}+c_{m}}+1\right)\left(\frac{\Lambda}{\theta_{h}+\mu_{h}}+1\right)\left(\frac{\Lambda}{\mu_{m}+c_{m}}+1\right)\left(\frac{\Lambda}{\alpha_{h}+\mu_{h}}+1\right) .
$$

Therefore, plotting the number of new cases per week (weekly incidence) against the cumulative number of cases, the phase of exponential growth of the cumulative number of cases is evidenced by a linear growth of the curve, whose slope is the force of infection (Figure 1), and can be computed by a least-square linear fit of this linear phase [4]. For the data of the 1995 dengue outbreak in the city of Salvador, Bahı, Brazil shown in Figure 1, we obtain $\Lambda=0.43 \pm 0.02$ weeks ${ }^{-1}$. Using the average temperature record in the same year at Salvador, $T=25.8 \pm 0.4^{\circ} \mathrm{C}$ (given by...), the mosquito entomological parameters are $\mu_{m}=0.0302$ days $^{-1}$ (with minimum and maximum values relative to the mean temperature range of 0.0296 and 0.0308 days $^{-1}$ ), and $\theta_{m}=0.0957$ days $^{-1}$ (with minimum and maximum values relative to the mean temperature range of 0.0938 and 0.0977 days $^{-1}$ ) [8]. The human mean parameters are $\alpha_{h}=0.125$ days $^{-1}$, $\mu_{h}=4 \times 10^{-5}$ days $^{-1}$ and $\theta_{h}=0.17$ days $^{-1}$. Substituting these parameter values and $c_{m}=0$ (no vector control was in course during this epidemic) in equation 7 we obtain an estimation of $R_{0}=2.85$ (with minimum and maximum values relative to the human parameter's range (Table 1) of $R_{0}=2.77$ and 3.70$)$.

Using the same equation, we measured the control efforts, represented by the parameter $c_{m}$, necessary to reduce dengue incidence (Figure 2), i.e., to diminish $R_{0}<1$. Observe that control mechanisms, applied to mosquito adult form during dengue outbreak, cannot reduce $R_{0}$ to values smaller than one, and therefore, these mechanisms are not sufficient to contain the dengue epidemic. However, Figure 2 shows that control efficacy is greater for $c_{m}<0.2$ and, since control strategies have a cost for governmental authorities, there is no doubt that developing theoretical and experimental techniques to identify the optimal control strategy is a central challenge in dengue mosquito control. The results above make evident that other mechanisms, related to the control of the aquatic phase, are necessary to avoid dengue outbreaks, reducing the force of infection before its triggering.

In the case of Salvador's dengue outbreak occurred in 2002, control mechanisms applied to adult mosquito population were applied, mostly in places where high number of classic, and principally dengue hemorrhagic was notified. Therefore, for the data of the 2002 dengue outbreak in the city of Salvador, Bahı, Brazil, shown in Figure 3, we obtain $\Lambda=0.38 \pm 0.02$ 


\section{Anals do GNIVAG $\mathrm{V}, 3$

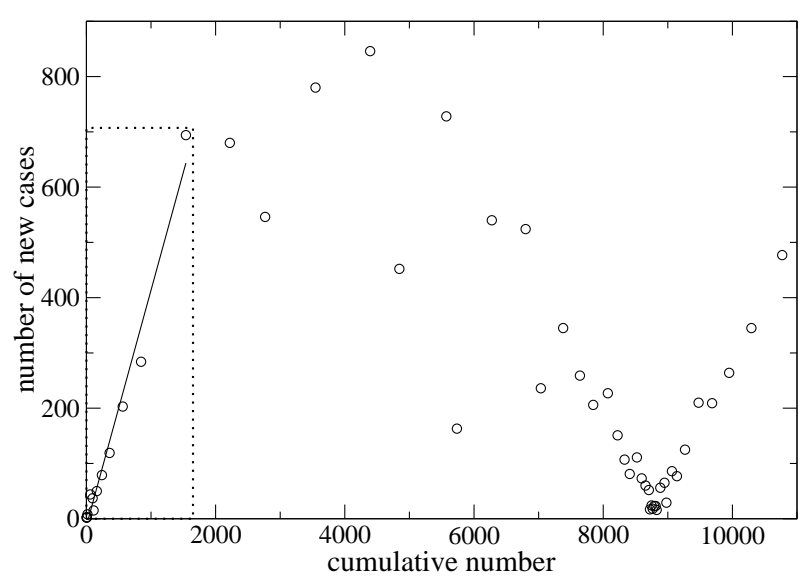

Figura 1: Weekly number of cases against the cumulative number of cases for dengue outbreak in the city of Salvador in 1995-1996. The dashed box indicates the growing linear parts of the plots corresponding to the initial exponential growth of the epidemics. The least square linear fit of the linear phase gives $\Lambda=0.43 \pm 0.02$.

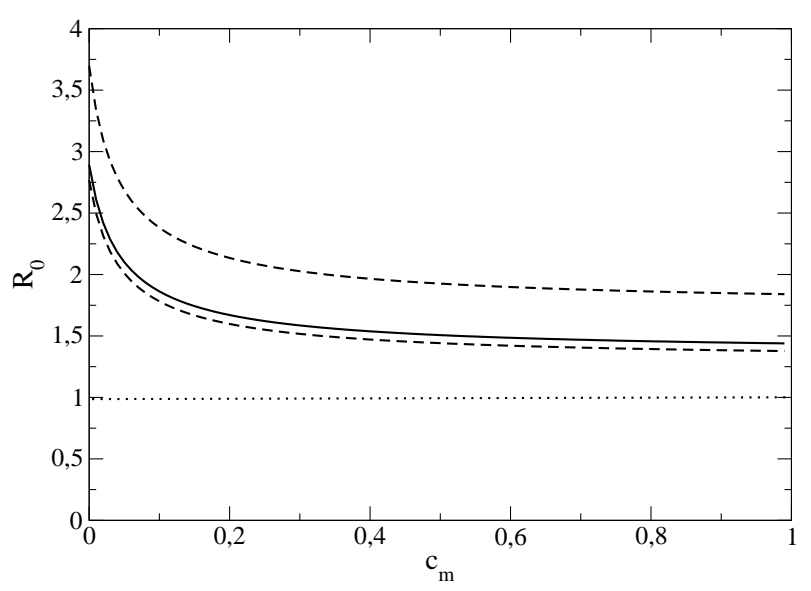

Figura 2: Value of $R_{0}$ for dengue outbreak at Salvador in 1995 as a function of control efforts. The continuous line represent the average model's output and the dashed line the maximum and minimum value obtained using the minimum and maximum values relative to the human parameter's range. 


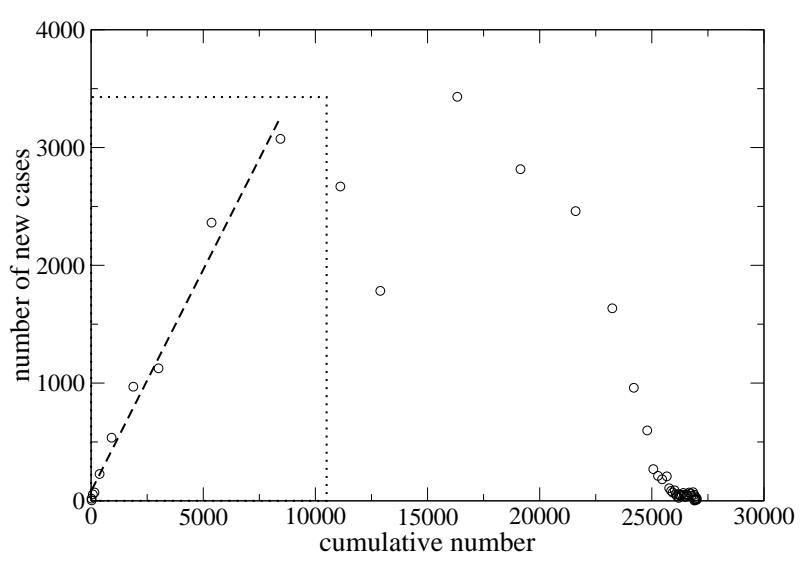

Figura 3: Weekly number of cases against the cumulative number of cases for dengue outbreak in the city of Salvador at 2002. The dashed box indicate the growing linear parts of the plots corresponding to the initial exponential growth of the epidemics. The least square linear fit of the linear phase gives $\Lambda=0.38 \pm 0.02$.

weeks ${ }^{-1}$. For the temperature data recorded in 2002 we calculated an average temperature of $T=25.6 \pm 0.4^{\circ} \mathrm{C}$ similar to that obtained in 1995, therefore, the estimated valued for the mosquito entomological parameters are the same. Comparing Figures 1 and 3, we observe that the force of infection is smaller for the dengue outbreak occurred in 2002. Hence, the vector control applied by Salvador's governmental authorities in this year, reduced by $12 \%$ the dengue's infection force avoiding a second positive slope similar to that one occurred in 1996 (see Figure 1) which maintained dengue in an endemic situation during 2003.

\section{Effective reproductive number}

When an epidemic started in a partially susceptible population or control measures of the disease have been implemented, it is more convenient to work with the effective reproductive number, $R(t)$, defined as the number of secondary infections arising from a primary case with symptoms onset at day $t$. In such conditions, the assumption of a constant environment and exponential increase of new cases is untenable. The value of $R(t)$ is an indication of the severity of the epidemic, and give information about the measures needed to control the disease.

Estimates for $R(t)$ can be obtained from the incidence curve of the human infectives using the following equation derived from the renewal equation for a birth process

$$
F(t)=\frac{b(t)}{\int_{a=0}^{\infty} b(t-a) g(a) d a}
$$

where in the epidemiological context, $b(t)$ accounts for the number of new cases at day $t$, and $g(a)$ is the generation interval distribution for the disease, which is defined as the probability distribution function for the time from infection of an individual to the infection of a secondary case by that individual.

In this section, we apply the method given in [11] to the model (1) using data of the 1995 and 2002 dengue outbreaks in the city of Salvador. The developed model (1) have exposed and infective stages both in human and mosquito populations. The rates of leaving the exposed and infectious classes are constant and denoted by $s_{1}=\theta_{m}+\mu_{m}+c_{m}, s_{2}=\mu_{m}+c_{m}, s_{3}=$ $\theta_{h}+\mu_{h}, s_{4}=\alpha_{h}+\mu_{h}$, respectively. Therefore, the generation interval distribution is the convolution of the four exponential distributions $s_{1} e^{-s_{1} t}, s_{2} e^{-s_{2} t}, s_{3} e^{-s_{3} t}, s_{4} e^{-s_{4} t}$ with a mean 


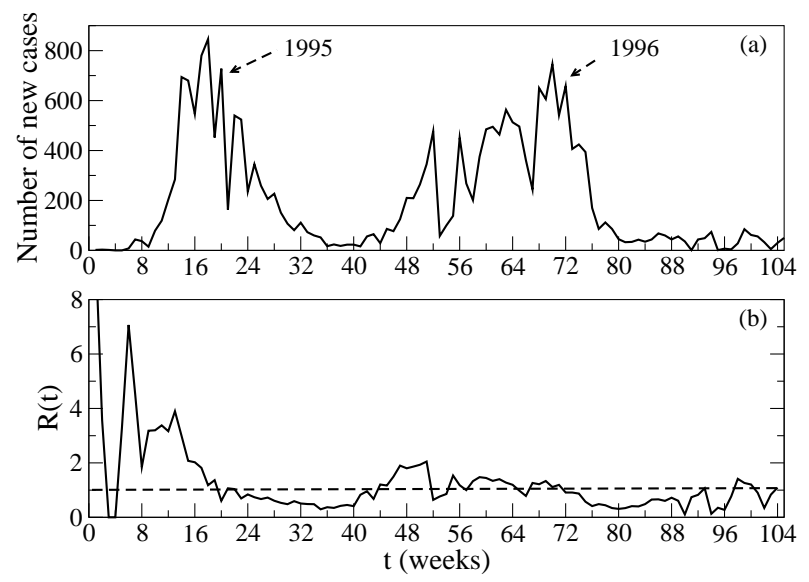

Figura 4: In (a) dengue cases during 1995-1996 and (b) the effective renroductive number $R(t)$ versus $t$.

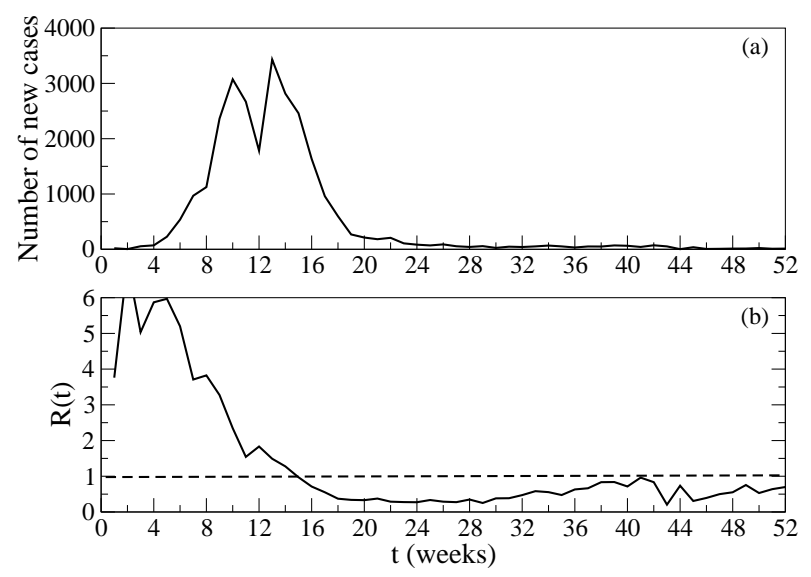

Figura 5: (a) dengue cases during 2002, (b) the effective reproductive number $R(t)$ versus $t$.

$T_{c}=1 / s_{1}+1 / s_{2}+1 / s_{3}+1 / s_{4}$ given by

$$
g(t)=\Sigma_{i=1}^{4} \frac{s_{1} s_{2} s_{3} s_{4} e^{-s_{i} t}}{\prod_{j=1, j \neq i}^{4}\left(s_{j}-s_{i}\right)}
$$

with $t \geq 0$ [1]. The relations above are valid when the infection force, $\Lambda$, satisfies the following relation: $\Lambda>\min \left(-s_{1},-s_{2},-s_{3},-s_{4}\right)$ [11]. Also, as we are dealing with distribution, $\int_{0}^{\infty} g(t) d t=1$.

Substituting $g(t)$ into equation (8) and using the epidemiological data of Figures 4 and 5, we compute $F(t)$, and since the number of human secondary cases derived from a human primary case is equal to $R^{2}(t)$, then $R(t)=\sqrt{F(t)}$.

Figures $4 \mathrm{a}$ and $5 \mathrm{a}$ show the time evolution of the weekly incidence for the dengue outbreak in the city of Salvador in 1995-96 and 2002, respectively. Although the incidence peak in 2002 was larger than in 1995, vector control measures applied in 2002 were able to avoid a secondary dengue outbreak in 2003. Furthermore, comparing Figures $4 \mathrm{~b}$ and $5 \mathrm{~b}$ with respect to the tangent slopes measured during the interval of disease decreasing (after the maximum weekly incidence), we see that the rapid and effective strategies applied by the governmental authorities to control dengue disease at 2002 were able to reduce the epidemic period by a half, driven the disease from an epidemic situation to an endemic state ten times faster than in the 1996 outbreak when the return to an endemic situation was made naturally by the short and long term protection from 
infection, so that the depletion of the human susceptible pool brings the epidemic to a halt. Indeed, the highest dengue incidence observed at 2002 was associated to the virus virulence, DENV3, which was greater than the DENV2 virulence responsible of the outbreak of 1995.

Moreover, analyzing Figure $4 \mathrm{~b}$, we observe that $R(t)$ assumes values greater than one at the end of 1995 promoting a second dengue outbreak at 1996 when the temperature and rainfall favored the proliferation of the mosquito $A$. aeypti, and in consequence, dengue transmission. On the contrary, Figure 5b shows that in2002 the value of $R(t)$ does not assume values greater than one after the first decrease corroborating the endemic dengue situation observed at 2003.

\section{ACKNOWLEDGMENTS}

The authors acknowledge the Brazilian agencies CNPq and FAPESB for financial support; G. Ribeiro and INMET for temperature data. L. Esteva acknowledges FENOMEC and Grant IN108607-3 of PAPIIT-UNAM and Ferreira acknowledges FAPESP 2010/01489-5.

\section{Referências}

[1] Akkouchi, M. (2008). On the convolution of exponential distributions. J. of the Chungcheong Math. Soc. 21: 501-510.

[2] Diekmann, O. and Heesterbeek J. A. P. (2000). Mathematical Epidemiology of Infectious Diseases: Model Building, Analysis and Interpretation. Wiley, New York.

[3] Esteva, L. and Yang, H. M. (2005). Mathematical Model to Assess the Control of Aedes aegypti Mosquitoes by the Sterile Insect Technique. Math. Biosci. 198: 132-147.

[4] Favier, C., Degallier, N., Rosa-Freitas, M. G., Boulanger, J. R. , et al. (2006). Early determination of the reproductive number for vector-borne diseases: the case of dengue in Brazil. Trop.Med. and Int. Health 11 (3): 332-340.

[5] Focks, D.A., Haili, D. G., Daniels, E., and Mount, G. A. (1993). Dynamic life table model of or Aedes aegypti (Dipetera: Culicidae): analysis of the literature and model development. J. of Med. Ent. 30: 1003-1017.

[6] Van den Driessche, P. and J. Watmough (2002). Reproduction numbers and sub-threshold endemic equilibria for compartmental models of disease transmission. Math. Biosci. 180: $29-48$.

[7] Yang, H.M., Macoris, M. L. G., Galvani, K.C., Andrighetti, M. T. M., and Wanderley, D.M.V. (2009). Assessing the effects of temperature on dengue transmission. Epidemiol. Infect., 137(8): 1179-1187.

[8] Yang, H.M., Macoris, M. L. G., Galvani, K.C., Andrighetti, M. T. M., and Wanderley, D.M.V. (2009). Assesing the effects of temperature on the population of Aedes aegypti, the vector of dengue. Epidemiol. Infect. 137: 1188-1202.

[9] Yang, H.M. \& Ferreira, C.P. (2008). Assessing the effects of vector control on dengue transmission. Applied Mathematics and Computation, 198:401-413.

[10] Ferreira, C.P. ; Yang, H M ; Esteva, L. (2008). Assessing the suitability of sterile insect technique applied to aedes aegypti. Journal of Biological Systems, 16(4):1-13..

[11] Wallinga, J. and Lipsitch, M. (2007). How generation intervals shape the relationship between growth rates and reproductive numbers. Proc. R. Soc. B 274: 599-604 
[12] Newton E.A.C and Reiter P. (1992). A model of the transmission of dengue fever with an evaluation of the impact of ultra-low volume (ULV) insecticide applications on dengue epidemic. Am. J. Trop. Med. Hyg. 47(6): 709-720.

[13] Dibo, M.R.; Chierotti, A.P.; Ferrari, M.S.; Mendonça, A.L.; Neto, F.C. (2008). Study of the relationship between Aedes (Stegomyia) aegypti egg and adult densities, dengue fever and climate in Mirassol, state of São Paulo, Brazil. Mem Inst Oswaldo Cruz, Rio de Janeiro, 103(6): 554-560. 